\title{
The global epidemic of chronic kidney disease
}

\section{Author: M.R. Davids}

Division of Nephrology and Department of Medicine Stellenbosch University and Tygerberg Hospital

Address for correspondence:

Associate Professor M. R. Davids

Department of Medicine

Tygerberg Hospital

Tygerberg

7505 South Africa

\section{Email:}

mrd@sun.ac.za

ABSTRACT Chronic kidney disease (CKD) is defined as kidney damage or a glomerular filtration rate (GFR) of $<60 \mathrm{ml} /$ $\mathrm{min} / \mathrm{l} .73 \mathrm{~m}^{2}$ for 3 months or more. About one adult in ten, or over 500 million people worldwide, are affected, with diabetic nephropathy, glomerulonephritis and uncontrolled hypertension being the major causes. The global burden of CKD is expected to increase in parallel with the increase in diabetes, with the developing world bearing the brunt of this epidemic.

The major consequences of CKD are the development of end-stage renal disease (ESRD) and, even more frequently, premature death from cardiovascular disease. This results from a markedly increased prevalence as well as a higher case fatality rate. CKD is increasingly being recognised as a very strong risk factor for cardiovascular disease, justifying lower targets for blood pressure and lipid control. Apart from the frequent presence of traditional risk factors, these patients often suffer from nontraditional factors such as hyperphosphataemia, hyperhomocysteinaemia, malnutrition, chronic fluid overload and anaemia.

The management of ESRD is extremely costly, and it is therefore not surprising that most patients on treatment are to be found in high income countries. In many African countries there is little or no treatment available. The focus has therefore turned to the early detection and treatment of CKD to prevent progression to ESRD and to decrease the morbidity and mortality from cardiovascular disease. Several international initiatives have been launched in response to this global public health problem.

Chronic kidney disease (CKD) is defined as kidney damage or a glomerular filtration rate (GFR) of $<60 \mathrm{ml} / \mathrm{min} / 1.73 \mathrm{~m}^{2}$ for 3 months or more, irrespective of the cause.(I) Kidney damage may be indicated by the presence of abnormalities of the composition of blood or urine (e.g. proteinuria or albuminuria on random urine specimens), or by the presence of abnormalities on renal imaging; GFR should be estimated from serum creatinine using prediction equations like the Cockcroft Gault formula or the Modification of Diet in Renal Disease (MDRD) equation. It is worth noting that the diagnosis of CKD does not require 24-hour urine collections for creatinine clearance or protein quantification.
Patients with CKD are classified according to GFR (Table I), ranging from stages I and 2 where there are persistent urinary abnormalities but preserved GFR, through to stages 4 and 5, which represent advanced and end-stage renal disease (ESRD) respectively. In the early stages, the emphasis is on detection and prevention of progression, while during the later stages the focus includes the management of the complications of CKD and preparation for renal replacement therapy.

TABLE I: Definition and classification of chronic kidney disease»

\begin{tabular}{c|ll}
\hline Stage & \multicolumn{1}{|c}{ Description } & GFR in $\mathrm{ml} / \mathrm{min} / 1.73 \mathrm{~m}^{2}$ \\
\hline 1 & Kidney damage\# with normal or increased GFR & $\geq 90$ \\
\hline 2 & Kidney damage with mild decrease in GFR & $60-89$ \\
3 & Moderate decrease in GFR & $30-59$ \\
4 & Severe decrease in GFR & $15-29$ \\
\hline 5 & Kidney failure & $<15$ or dialysis \\
\hline
\end{tabular}

* Requires the presence of kidney damage or a decrease in GFR for $\geq 3$ months.

\# Indicated by the presence of abnormalities of the composition of blood or urine (e.g. proteinuria albuminuria, haematuria), or structural abnormalities. Patients found to have a GFR of 60-89 mll $\mathrm{min} / 1.73 \mathrm{~m}^{2}$ without one of these markers should not be considered to have CKD and need not be subjected to further investigation.

This standardised scheme for the detection and classification of CKD has been developed during the last few years and been universally accepted; it will do much to raise awareness of this important problem and is essential for the international development and implementation of clinical practice guidelines.

\section{THE MAGNITUDE AND CAUSES OF THE GLOBAL EPIDEMIC}

CKD affects as many as 1 in 10 adults, or over 500 million people worldwide. It is a worldwide public health problem which leads to progressive renal failure, cardiovascular disease and premature death. ${ }^{(2,3)}$ The Third National Health and Nutrition Examination Surveys (NHANES III) in the USA found that an estimated 20 million Americans have CKD. ${ }^{(4)}$ It is among the leading causes of death in the industrialised world, and the 9th most important cause of death in the USA.

Diabetic nephropathy is one of the leading causes of CKD and ESRD, and the global epidemic of obesity and type 2 diabetes will therefore result in millions of new cases of CKD. It is estimated that the number of people with diabetes will rise from 17I million in 2000 to 366 million in 2030. What is of particular concern is that the greatest burden will be borne by countries in the developing world, where there are usually 
scant resources available to deal with the problem. The largest relative increases will occur in the Middle East, sub-Saharan Africa and India. In absolute numbers, the countries with the largest projected numbers of cases in 2030 will be India (79.4 million), China (42.3 million) and the USA (30.3 million)..(5) It is therefore imperative that the focus be turned to the prevention of CKD, and the slowing of the progression of the early stages of CKD to ESRD with its serious and costly complications.

Chronic glomerulonephritis and interstitial nephritis cause most cases of chronic kidney disease in developing countries because of the high prevalence of infections. Streptococcal infections are common in Africa, while tuberculosis is a particular problem in causing CKD in the Middle East and India. In Africa the hepatitis B and C viruses, and increasingly HIV, are important causes of CKD. Parasitic infections may cause ureteric obstruction (e.g. schistosomiasis), interstitial nephritis (visceral leishmaniasis) and glomerulonephritis (malaria, filariasis, schistosomiasis). ${ }^{(6)}$

\section{EXCESS MORBIDITY AND MORTALITY FROM CARDIOVASCULAR DISEASE}

All patients with CKD, irrespective of the stage, have an increased risk of death from heart and cerebrovascular disease. (7) The relative risk of cardiovascular disease increases from about 1.5 among patients with isolated proteinuria to almost 500 among young patients on chronic dialysis. $(6,8)$ A patient with CKD is far more likely to succumb to cardiovascular disease than to progress to end-stage renal disease (ESRD) requiring dialysis or transplantation. In those who do reach ESRD and are included in dialysis and transplant programmes, CVD mortality is 10 to 30 times higher than in the general population, despite stratification for sex, race, and the presence of diabetes. ${ }^{(8)}$ The high mortality rate is likely due to both a high case fatality rate and a high prevalence of CVD. Mortality I and 2 years after myocardial infarction was $59 \%$ and $73 \%$ in dialysis patients, which is much higher than that expected in the general population, even in subjects with comorbid conditions such as diabetes. ${ }^{(9)}$ Anavekar et al. examined the impact of renal function on cardiovascular outcomes after myocardial infarction in over 14000 patients as part of the Valsartan in Acute Myocardial Infarction Trial (VALIANT). ${ }^{(10)}$ Decreasing GFRs were associated with increasing mortality rates (Figure I). Three-year mortality was $14.1 \%$ in the group with an estimated GFR (in $\mathrm{ml} / \mathrm{min} / 1.73 \mathrm{~m}^{2}$ ) of $\geq 75.0,20.5 \%$ with a GFR of $60.0-74.9,28.9 \%$ when GFR was $45.0-59.9$ and $45.5 \%$ when GFR was $<45.0$.

The tremendous burden of cardiovascular disease in patients with CKD is attributed to the frequent presence of traditional factors, such as hypertension and dyslipidemia, as well as to nontraditional factors such as hyperphosphataemia and soft tissue calcification, hyperhomocysteinaemia, inflammation, malnutrition, chronic fluid overload, anaemia and other conditions.

The presence of CKD, whether it is manifested by proteinuria or reduced glomerular filtration rate (GFR), is now acknowledged as an

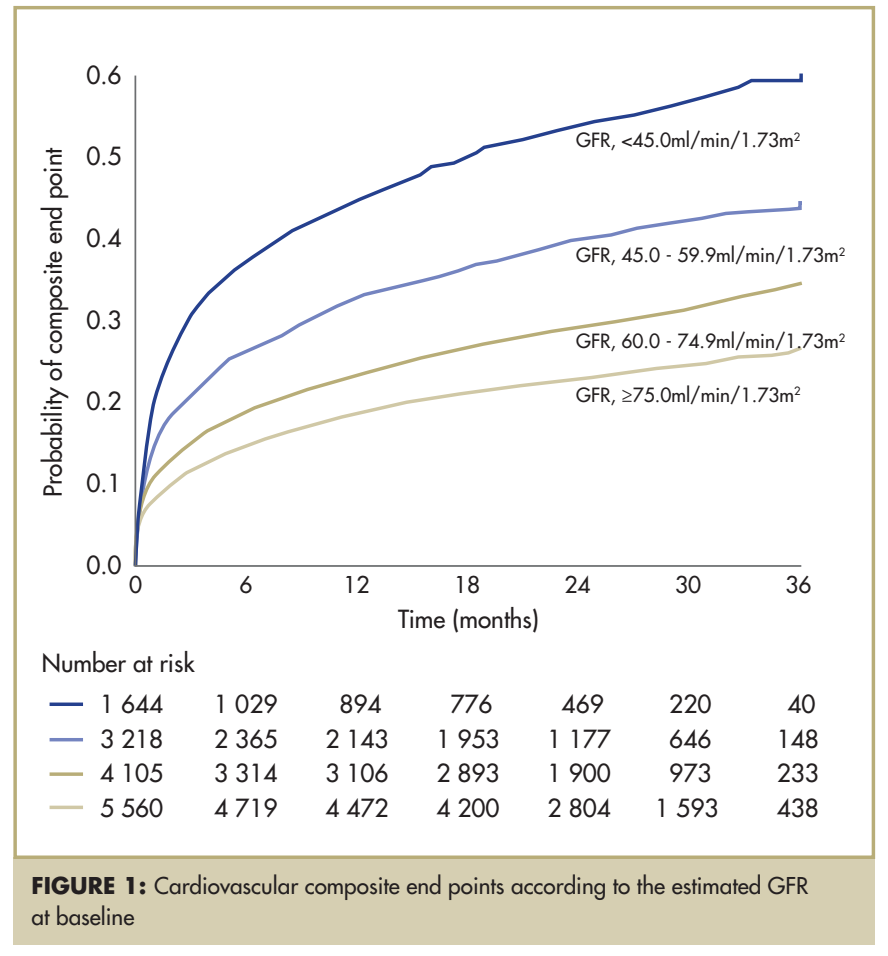

Modified from N Engl I Med 351: 1 285-95 Anavekar et al(10)

Copyright () 2004 Massachusetts Medical Society. All rights reserved.

independent risk factor for cardiovascular disease events (CVD) in the most recent report from the Joint National Committee on Prevention, Detection, and Treatment of High Blood Pressure (JNC VII) and in a position statement of the American Heart Association. (11, 12) Patients with CKD are considered to be in the highest-risk group by JNCVII and the US National Kidney Foundation, with JNC VII including CKD as a "compelling" indication for optimal blood pressure control, justifying lowertarget blood pressure and treatment with specific antihypertensive agents. Both the US National Kidney Foundation and the American Heart Association recommend that patients with CKD be included in the highest-risk group for treatment of dyslipidaemia, justifying a lower target low-density lipoprotein cholesterol level. (12)

\section{MALNUTRITION, INFLAMMATION AND ATHEROSCLEROSIS}

Protein-energy malnutrition and inflammation are common in patients with chronic kidney disease and worsen with progression toward endstage renal disease.These are major predictors of poor clinical outcome, as reflected by the strong association between hypoalbuminaemia and cardiovascular disease. Among dialysis patients, traditional indicators of overnutrition (high cholesterol or body mass index (BMI)), which are risk factors for cardiovascular disease in the general population, are associated with better outcomes, while a low BMI and low cholesterol or creatinine are risk factors for a poor outcome. These paradoxical relationships between nutritional markers and outcome are referred to as "reverse epidemiology". 
In the Dialysis Outcomes and Practice Patterns Study (DOPPS), which involves 12 countries, nutrition-related parameters associated with increased mortality included low BMI, low subjective global assessment (SGA) score, and hypoalbuminaemia. Mortality risk was increased 1.38 times with serum albumin levels below $35 \mathrm{~g} / \mathrm{l}$. In the US patient cohort there was a 2.12-fold increase of the relative risk of death for the lowest quartile (serum albumin $<33 \mathrm{~g} / \mathrm{l})$ compared with the highest quartile (>40 g/l).

It has been suggested that inflammation is the cause of both malnutrition and CVD. The terms malnutrition-inflammation complex syndrome (MICS) and malnutrition-inflammation-atherosclerosis (MIA) syndrome have been coined to indicate this interaction and the important contribution of both of these conditions to poor clinical outcome. . $^{(13-15)}$ Causes of inflammation in dialysis patients include, among others, exposure to dialysis membranes or peritoneal dialysis fluid, poor water quality, peritonitis, and other infections. So far no randomised clinical trials have specifically examined the effect of nutritional or antiinflammatory interventions on outcomes. It still remains unclear whether the association with worse outcomes is related to malnutrition at the time of initiation of dialysis or whether it is secondary to changes in nutritional status of ESRD patients over time. ${ }^{(16)}$

\section{TREATMENT FOR END-STAGE RENAL DISEASE}

Worldwide there are well over 1.3 million people on maintenance dialysis, and this number is projected to exceed 2 million by $2010 .{ }^{(17)}$ Haemodialysis (89\%) is much more common than peritoneal dialysis ( $11 \%)$ as the treatment modality. Japan has the highest prevalence rate of ESRD in the world, at I 857 per million population (pmp) in 2004 followed by Taiwan with I 706. In the USA 104364 new dialysis and transplant patients began ESRD therapy in 2004, an incidence of 339 pmp. More than 470000 patients were on treatment for ESRD in the USA on 31 December 2004, a prevalence rate of 1542 pmp 335963 were on dialysis, and 136 I 36 had a functioning transplant. ${ }^{(18)}$

As prevalence of ESRD is a function of incidence and deaths, and if we assume a similar annual incidence across most countries of the world (it is estimated at 150 per million population in developing countries) ${ }^{(6)}$ then the wide variation in prevalence mainly reflects differences in survival, which in turn results from the differences in the availability of dialysis and transplantation. This provision of renal replacement services (and prevalence of ESRD) correlates well with income per capita, especially in lower income countries. ${ }^{(17)}$

Given the costs of treating ESRD, it is not surprising that most of the world's dialysis patients are being treated in high income countries, with 52\% from just four countries: the USA, Japan, Brazil and Germany. Over $6 \%$ of the US Medicare budget is spent on ESRD, with total annual costs for ESRD reaching $\$ 32.5$ billion. Based on the US average of around $\$ 66000$ per patient per annum, it is estimated that I trillion dollars would be needed to care for ESRD patients worldwide from 2001 to $2010 .(19)$

Barsoum points out that renal replacement therapy represents an unaffordable financial burden for most poor countries, and often has negative psycho-social effects on communities in these countries. ${ }^{(6)}$ Frequently, patients undergoing chronic dialysis are only partially rehabilitated, and remain unable to work. Dialysis is often inefficient (due to resource limitations); essential treatments such as erythropoietin, intravenous iron, active vitamin D, and statins may not be available; coexisting infections and malnutrition are common; and transportation difficulties often result in noncompliance.

In countries in which the GDP per capita is below US\$10 000 there is a significant correlation between GDP and ESRD prevalence. In India and Pakistan, less than 10\% of patients with ESRD are offered renal replacement therapy and the majority of these stop treatment because of cost constraints within the first three months. ${ }^{(20)}$ Treatment rates in North Africa vary from 30 to 186.5 per million population where there are established programmes - Algeria 78.5, Egypt 129.3, Libya 30, Morocco 55.6, and Tunisia 186.5 pmp. Services are mainly available in the urban areas. In sub-Saharan Africa, a conservative approach to therapy most often applies. Few patients can afford chronic dialysis and renal transplantation is often not available.(21)

In South Africa, no current information is available on the numbers or the outcomes of patients with CKD or ESRD. The last formal report from the SA Dialysis and Transplant Registry was in 1994 and revealed a prevalence of 99 pmp (3 399 patients) with half of these being on dialysis and half having functioning grafts. ${ }^{(21)}$ Two-thirds of the patients on dialysis were on haemodialysis and one-third on peritoneal dialysis. It is interesting to examine the prevalence data of countries who have similar gross national incomes per capita (GNIPC) to our own - South Africa has a GNIPC of $\$ 4960$, Malaysia $\$ 4960$, Turkey $\$ 4710$ and Thailand $\$ 2$ 750. In 2004 Malaysia had an ESRD prevalence rate per million of 522 (total 13348 patients), Turkey 433 (3I 25 I), and Thailand 243 (15 083).(18) There are no comparable data available for South Africa; however, the national Dialysis and Transplant Registry is currently being reestablished as a joint initiative of the South African Renal and Transplantation Societies.

In Malaysia, where renal replacement therapy has seen an exponential growth since 1990,(22) this increase has been coupled to the growth in the country's income per capita. In recent years greater numbers of diabetics (5I\% of new patients in 2003) and older patients have been admitted to their programme. It would seem reasonable that the resources made available for renal replacement therapy in the public sector in South Africa be linked to our GNIPC and that, at the present time, a prevalence rate of 200-300 pmp could be expected. 


\section{TREATING CKD: POOR OUTCOMES AND VARIATIONS IN CLINICAL PRACTICE}

There is convincing evidence that the adverse complications of CKD can be prevented or delayed by effective treatment of the earlier stages of CKD, using strategies that include smoking cessation, salt restriction, increasing physical activity, maintenance of a healthy weight, reduction in proteinuria, good blood pressure control and blockade of the reninangiotensin system (Figure 2).(23-25)

Many studies have confirmed that ACE inhibition or the use of angiotensin receptor blockers is protective through combined antihypertensive and antiproteinuric effects. The group of Remuzzi(26) has suggested that aggressive medical treatment to achieve multifactorial risk factor reduction can normalize or even reverse the annual rate of loss of renal function. Wendy Hoy, working with an Australian Aboriginal community, showed marked reductions in natural deaths due to renal and cardiovascular causes after instituting a prevention and treatment programme. ${ }^{(27)}$

There is, however, much variability in the translation of research findings to improvements in clinical practice. Within the nephrology community, differences in renal patient survival in different countries came to the fore in 1989 at the Dallas symposium on morbidity and mortality of dialysis patients, with the USA recording the highest all-cause annual mortality (22-24\%). ${ }^{(28)}$ For a patient with CKD in the USA, the outcome was worse than for an equivalent patient with a diagnosis of colon or prostate cancer. ${ }^{(29)}$ It was thought that differences in practice patterns could be responsible for these differences in outcomes. The Third
National Health and Nutrition Examination Surveys (NHANES III) in the USA revealed that only $27 \%$ of patients with CKD had a blood pressure $<140 / 90 \mathrm{mmHg}$, and a majority of patients had severe anaemia (mean haematocrit 27.7\%), with only one-quarter being prescribed erythropoietin despite insurance cover being available for most of them. Despite the clear benefits of renal transplantation, most eligible patients had not been placed on a transplant waiting list 6 months after beginning dialysis. Lastly, more than $50 \%$ of patients on the US ESRD programme were considered malnourished. ${ }^{(29)}$

While we lack good data for South Africa, there is no reason to believe that we do not have similar problems, with failure to reach therapeutic targets and lack of awareness of clinical practice guidelines, or with guidelines not being effectively implemented. ${ }^{(30)}$ We must continue to strive for the successful implementation of rigorously developed evidence-based clinical practice guidelines, as this offers a way of reducing variability of care and improving patient outcomes.

\section{EVIDENCE-BASED GUIDELINES AND INITIATIVES TO IMPROVE GLOBAL OUTCOMES}

Given that there is general consensus that CKD represents a major global public health problem, effective action is required at national and international levels, and cooperation across disciplines is essential. Several such initiatives are briefly described below, which represent an encouraging response to this urgent call to action.

\section{K/DOQI}

In 1999 the Kidney Disease Outcomes Quality Initiative (K/DOQI) was launched by the US National Kidney Foundation - broadening the
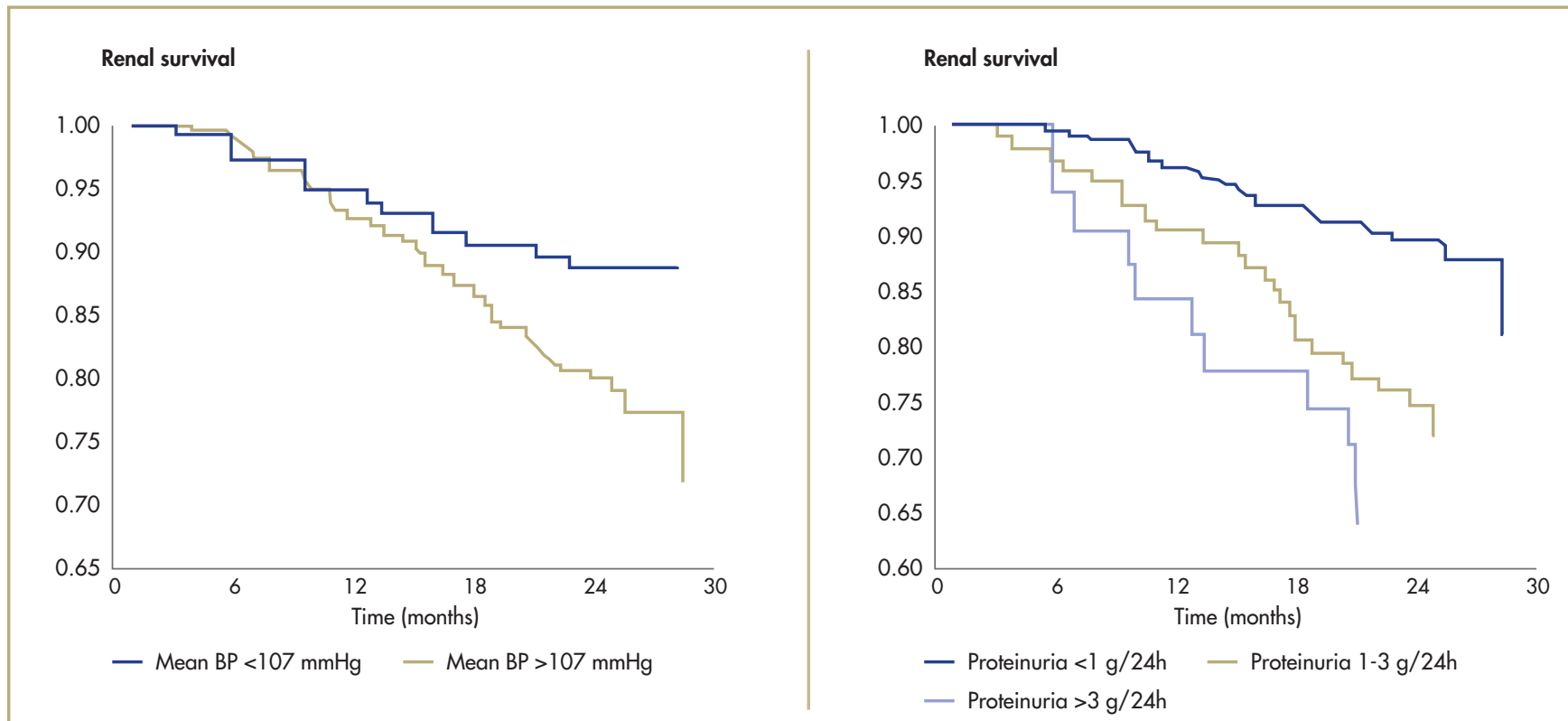

FIGURE 2: Effect of BP and proteinuria on renal survival

Reprinted from Nephrol Dial Transplant 1 1:46 I -7 Locatelli et al. 23 (1996) with permission. 
scope of an earlier project to include all patients with kidney disease, from the earliest stages of kidney damage to end-stage kidney failure requiring dialysis and transplantation. The European Renal AssociationEuropean Dialysis and Transplantation Association have produced the European Best Practice Guidelines, and clinical practice guidelines have also been developed by the Canadian Society of Nephrology, the United Kingdom Renal Association, and the Australian and New Zealand Society of Nephrology. ${ }^{(2)}$

While there may be regional differences in risk factors and in available resources, the complications and problems of patients with kidney disease are universal. It is increasingly being felt that a uniform, global and multi-disciplinary approach to developing and implementing clinical practice guidelines is required.

\section{KDIGO}

A new global organisation, Kidney Disease: Improving Global Outcomes (KDIGO), has been formed in an attempt to "improve the care and outcomes of kidney disease patients worldwide through promoting coordination, collaboration and integration of initiatives to develop and implement clinical practice guidelines" (2). The KDIGO board of directors includes world leaders in nephrology, with representation from North and South America, Europe, Africa and the Far East. Different work groups will focus on matters such as (i) a uniform system of evidence rating, (ii) the adoption of a common evaluation, classification and nomenclature for CKD worldwide, (iii) the establishment of a database of currently available guidelines, (iv) the implementation of guidelines, (v) development efforts in regions without guidelines, and (vi) fostering coordination between K/DOQI and the European Best Practice Guidelines.

\section{KHDC}

The International Society of Nephrology (ISN) has designed a programme for the detection and management of kidney disease, hypertension, diabetes and cardiovascular disease (KHDC) in developing countries. The aims are to identify the individuals with these diseases by community-based or selective screening programmes and then to provide medical management including health education, lifestyle modification and pharmacological treatment in order eventually to reduce mortality and end-stage kidney disease. The documentation can be downloaded from the ISN website at http://www.nature.com/isn/ education/guidelines/isn/full/ed_05 I027_2.html.

\section{ISN}

The International Society of Nephrology provides funding for research and prevention programmes, and through its Commission for the Global Advancement in Nephrology (COMGAN) has been running a very successful fellowship programme which provides nephrological training at advanced institutions to physicians from emerging countries, with the ultimate goal of improving the standards in the Fellows' home countries upon their return. Through their Continuing Medical Education
(CME) programme, COMGAN reaches some 14000 doctors and health care practitioners in the emerging world annually. Their Sister Renal Center programme was launched in 1997 to enhance cooperation between nephrology centers worldwide. It aims to advance the practice of nephrology in the developing world by linking renal units there with centers of excellence. Other notable initiatives by the ISN include the provision of travel grants to major congresses, a Visiting Scholars programme which enables senior physicians and/or scientists to spend 6 weeks to 3 months at an institution in the developing world, and a Library Enhancement programme.

\section{The Bellagio 2004 Declaration}

In 2004 the International Society of Nephrology convened a multidisciplinary group of expert physicians and public health leaders from around the world to develop strategies to address CKD based on awareness, early detection and effective treatment. ${ }^{(31)}$ There was representation from the World Heart Federation, International Diabetes Federation, International Atherosclerosis Federation, and the International Society of Hypertension. Global perspectives on diabetes and cardiovascular and renal diseases were presented, with special emphasis on China, India, Latin America and Africa. Delegates agreed that an integrated global approach to chronic vascular diseases is needed and resolved to establish a 5-year plan for the prevention and treatment of chronic vascular diseases, including public advocacy, advising international and national agencies, and improving education and the practice of established approaches.

\section{The 2004 WHO report on Preventing Chronic Diseases}

In this report ${ }^{(32)}$ the $\mathrm{WHO}$ emphasises the fact that the burden of chronic disease will be borne mainly by low and middle income countries. They point out that there is no excuse for allowing chronic diseases to continue taking millions of lives each year when the scientific understanding of how to prevent these deaths is available now. Bold action is required, especially in the context of competing priorities.

\section{CONCLUSION}

It is important that we, in the developing world, do not fall into the trap of thinking that all our energies and resources should be poured into fighting infectious diseases like HIVIAIDS, tuberculosis and malaria. The data is clear that chronic, non-communicable diseases represent a terrible (and increasing) burden, causing 60\% of deaths across the world. Of these deaths, $80 \%$ occur in middle and lower income countries, home to most of the world's population. ${ }^{(32)}$

The scientific knowledge to address these challenges is already available. Measures to achieve prevention, or control the early stages of disease, are inexpensive and effective. However, they require large, coordinated, national programmes. Government "buy-in" and cooperation across our various medical disciplines is, therefore, essential if we are to prevent millions of premature deaths from heart disease, stroke, ESRD and diabetes. 


\section{REFERENCES:}

I. Levey AS, Eckardt KU,Tsukamoto Y, et al. Definition and classification of chronic kidney disease: a position statement from Kidney Disease: Improving Global Outcomes (KDIGO). Kidney Int 2005;67(6):2089-100

2. Eknoyan G, Lameire N, Barsoum R, et al.The burden of kidney disease: improving global outcomes. Kidney Int 2004;66(4): 1310-4.

3. Weisburger $\mathrm{JH}$. Worldwide prevention of cancer and other chronic diseases based on knowledge of mechanisms. Mutat Res 1998;402(1-2):331-7.

4. Coresh J, Astor BC, Greene T, Eknoyan G, Levey AS. Prevalence of chronic kidney disease and decreased kidney function in the adult US population:Third National Health and Nutrition Examination Survey. Am J Kidney Dis 2003;4 I ( I): I - 12.

5. Wild S, Roglic G, Green A, Sicree R, King H. Global prevalence of diabetes: estimates for the year 2000 and projections for 2030. Diabetes Care 2004;27(5): 1047-53.

6. Barsoum RS. Chronic kidney disease in the developing world. N Engl J Med 2006;354(10):997-9.

7. Levey AS, Beto JA, Coronado BE, et al. Controlling the epidemic of cardiovascular disease in chronic renal disease: what do we know? What do we need to learn? Where do we go from here? National Kidney Foundation Task Force on Cardiovascular Disease. Am J Kidney Dis 1998;32(5):853-906.

8. Foley RN, Parfrey PS, Sarnak MJ. Clinical epidemiology of cardiovascular disease in chronic renal disease. Am J Kidney Dis 1998;32(5 Suppl 3):SI 12-9.

9. Herzog CA, Ma JZ, Collins AJ. Poor long-term survival after acute myocardial infarction among patients on long-term dialysis. N Engl J Med 1998;339(I2):799-805.

10. Anavekar NS, McMurray J], Velazquez EJ, et al. Relation between renal dysfunction and cardiovascular outcomes after myocardial infarction. N Engl J Med 2004;35 I ( 3): 1285-95.

I I. Chobanian AV, Bakris GL, Black HR, et al. Seventh report of the Joint National Committee on Prevention, Detection, Evaluation, and Treatment of High Blood Pressure. Hypertension 2003;42(6): 1206-52.

12. Sarnak MJ, Levey AS, Schoolwerth AC, et al. Kidney disease as a risk factor for development of cardiovascular disease: a statement from the American Heart Association Councils on Kidney in Cardiovascular Disease, High Blood Pressure Research, Clinical Cardiology, and Epidemiology and Prevention. Circulation 2003; 108(17):2154-69.

13. Kaysen GA, Kumar V. Inflammation in ESRD: causes and potential consequences. J Ren Nutr 2003; | 3(2): | 58-60.

14. Kalantar-Zadeh K, Stenvinkel P, Pillon L, Kopple JD. Inflammation and nutrition in rena insufficiency. Adv Ren Replace Ther 2003; 1 0(3): 155-69.

15. Kaysen GA. Inflammation: cause of vascular disease and malnutrition in dialysis patients. Semin Nephrol 2004;24(5):431-6.

16. Byham-Gray LD. Outcomes research in nutrition and chronic kidney disease: perspectives, issues in practice, and processes for improvement. Adv Chronic Kidney Dis 2005; 12(1):96-106

17. Grassmann A, Gioberge S, Moeller S, Brown G. ESRD patients in 2004: global overview of patient numbers, treatment modalities and associated trends. Nephrol Dial Transplant 2005;20(I2):2587-93.

18. U.S. Renal Data System 2006 Annual Data Report: Atlas of End-Stage Renal Disease in the United States. Bethesda, MD: National Institutes of Health, National Institute of Diabetes and Digestive and Kidney Diseases; 2006.

19. Lysaght MJ. Maintenance dialysis population dynamics: current trends and long-term implications. J Am Soc Nephrol 2002; 13 Suppl I:S37-40

20. Sakhuja V, Sud K. End-stage renal disease in India and Pakistan: burden of disease and management issues. Kidney Int Suppl 2003(83):SI I5-8.

21. Naicker S. End-stage renal disease in sub-Saharan and South Africa. Kidney Int Suppl 2003(83):SI 19-22.

22. Hooi LS, Wong HS, Morad Z. Prevention of renal failure: the Malaysian experience. Kidney Int Suppl 2005(94):S70-4
23. Locatelli F, Marcelli D, Comelli M, et al. Proteinuria and blood pressure as causal components of progression to end-stage renal failure. Northern Italian Cooperative Study Group. Nephrol Dial Transplant 1996; I (3):46 I-7.

24. Campbell RC, Ruggenenti P, Remuzzi G. Halting the progression of chronic nephropathy J Am Soc Nephrol 2002;13 Suppl 3:S I90-5.

25. Taal MW, Brenner BM. Renoprotective benefits of RAS inhibition: from ACEl to angiotensin II antagonists. Kidney Int 2000;57(5): I 803-17.

26. Ruggenenti P, Schieppati A, Remuzzi G. Progression, remission, regression of chronic renal diseases. Lancet 200 I;357(9268): 1601-8

27. Hoy WE, Wang Z, Baker PR, Kelly AM. Secondary prevention of renal and cardiovascular disease: results of a renal and cardiovascular treatment program in an Australian aboriginal community. J Am Soc Nephrol 2003; I 4(7 Suppl 2):SI78-85.

28. Hull A, Parker T. Proceedings from the morbidity, mortality and prescription of dialysis symposium, Dallas Texas. September 15-17, 1989. Am J Kidney Dis 1990; 15:365-83.

29. Owen WF, Jr. Patterns of care for patients with chronic kidney disease in the United States: dying for improvement. J Am Soc Nephrol 2003; I 4(7 Suppl 2):S76-80

30. KalkWJ,Veriawa Y, Osler C.A survey of hospital outpatient services for chronic diseases in Gauteng. S Afr Med J 2000;90(I):57-61.

31. Dirks JH, de Zeeuw D, Agarwal SK, et al. Prevention of chronic kidney and vascular disease: toward global health equity - the Bellagio 2004 Declaration. Kidney Int Supp 2005(98):SI-6.

32. Preventing chronic diseases: a vital investment:World Health Organisation; 2004 